\title{
GW23-e2121 A PROTECTIVE FUNCTION OF THE NRF2 DURING HYPOXIA PRECONDITIONING INCREASING CELL SURVIVAL AND THE THERAPEUTIC POTENTIAL OF ENDOTHELIAL PROGENITOR CELLS
}

doi:10.1136/heartjnl-2012-302920a.51

Ranzun Zhao, Jian Feng, Guoxiang He, Guoxiang He. The Third Military Medical University

Objectives Accumulating evidence indicates that transient hypoxic preconditioning improves resistance to severe hypoxia and enhances the therapeutic potential of endothelial progenitor cells (EPCs) in cell-based therapies for vascular repair and ischaemic disease; however, the mechanisms underlying this process remain unclear. Nrf2 (nuclear factor E2-related factor 2) is a key transcription regulator that orchestrates protective cellular responses to oxidative stress. The objective of this study was to test the hypothesis that hypoxic preconditioning activates Nrf2 and the expression of its target gene, resulting in improved biological function and resistance to hypoxia.

Methods We observed that exposure to hypoxia following siRNAmediated knockdown of Nrf2 resulted in an increase in apoptosis, the inhibition of proliferation and impaired angiogenesis in vitro. Furthermore, hypoxic conditions activated Nrf2 via the PI3K/Akt signalling pathway, which resulted in the nuclear translocation of Nrf2 from the cytoplasm and a subsequent increase in the expression of one of the most important Nrf2 target genes: hame oxygenase 1 (HO-1).

Results We observed that exposure to hypoxia following siRNAmediated knockdown of Nrf2 resulted in an increase in apoptosis, the inhibition of proliferation and impaired angiogenesis in vitro. Furthermore, hypoxic conditions activated Nrf2 via the PI3K/Akt signalling pathway, which resulted in the nuclear translocation of Nrf2 from the cytoplasm and a subsequent increase in the expression of one of the most important Nrf2 target genes: hame oxygenase 1 (HO-1).

Conclusions EPCs exhibit resistance to hypoxia and improved therapeutic potential during hypoxia preconditioning that is mediated by the PI3K/Akt-Nrf2-HO-1 signalling pathway 